\title{
Coronary plaque redistribution after stent implantation is determined by lipid composition: A NIRS-IVUS analysis
}

Tomasz Roleder ${ }^{1}$, Magdalena Dobrolinska ${ }^{2}$, Elzbieta Pociask ${ }^{3}$, Wojciech Wanha ${ }^{2}$, Grzegorz Smolka ${ }^{2}$, Wojciech Walkowicz ${ }^{1}$, Radoslaw Parma ${ }^{2}$, Mariusz Lebek ${ }^{1}$, Tomasz Bochenek ${ }^{4}$, Przemyslaw Pietraszewski ${ }^{5}$, Elvin Kedhi ${ }^{6}$, Andrzej Ochala ${ }^{2}$, Zbigniew Gasior ${ }^{1}$, Ziad A. Ali ${ }^{7,8}$, Wojciech Wojakowski ${ }^{2}$

${ }^{1}$ Department of Cardiology, School of Health Sciences, Medical University of Silesia in Katowice, Poland ${ }^{2}$ Division of Cardiology and Structural Heart Diseases, Medical University of Silesia in Katowice, Poland ${ }^{3}$ Department of Biocybernetics and Biomedical Engineering,

AGH University of Science and Technology, Krakow, Poland

${ }^{4}$ First Division of Cardiology, Medical University of Silesia in Katowice, Poland

${ }^{5}$ Department of Sports Theory, Jerzy Kukuczka Academy of Physical Education in Katowice, Poland

${ }^{6}$ Isala Hartcentrum, Zwolle, Netherlands

${ }^{7}$ Center for Interventional Vascular Therapy, Division of Cardiology,

Presbyterian Hospital and Columbia University, New York, United States

${ }^{8}$ Cardiovascular Research Foundation, New York, United States

\section{Abstract}

Background: The composition of plaque impacts the results of stenting. The following study evaluated plaque redistribution related to stent implantation using combined near-infrared spectroscopy and intravascular ultrasound (NIRS-IVUS) imaging.

Methods: The present study included 49 patients (mean age $66 \pm 11$ years, $75 \%$ males) presenting with non-ST elevation myocardial infarction (8\%), unstable angina (49\%) and stable coronary artery disease (43\%). The following parameters were analyzed: mean plaque volume (MPV, $\mathrm{mm}^{3}$ ), plaque burden $(P B, \%)$, remodeling index (RI), and maximal lipid core burden index in a $4 \mathrm{~mm}$ segment $\left(\right.$ maxLCBI $\left._{4 m m}\right)$. High-lipid burden lesions $(H L B)$ were defined as by maxLCBI ${ }_{4 m m}>265$ with positive RI. Otherwise plaques were defined as low-lipid burden lesions (LLB). Measurements were done in the target lesion and in $4 \mathrm{~mm}$ edges of the stent before and after stent implantation.

Results: $M P V$ and maxLCBI ${ }_{4 m m}$ decreased in both HLB (MPV 144.70 [80.47, 274.25] vs. 97.60 [56.82, 223.45]; maxLCBI mmn $_{1}$ 564.11 \pm 166.82 vs. $\left.258.11 \pm 234.24, p=0.004\right)$ and LLB (MPV: 124.50 [68.00, 186.20] vs. 101.10 [67.87, 165.95]; $\operatorname{maxLCBI}_{4 m m}: 339.07 \pm 268.22$ vs. $124.60 \pm 160.96, p<0.001$ ), but MPV decrease was greater in HLB $(28.00$ [22.60, 57.10] vs. $13.50[1.50,28.84], p=0.019)$. Only at the proximal stent edge of $L L B$, maxLCBI $_{4 m m}$ decreased $(34[0,207]$ vs. $0[0,45], p=0.049)$ and plaque burden increased $(45.48[40.34,51.55]$ vs. $51.75[47.48,55.76], p=0.030)$.

Conclusions: NIRS-IVUS defined HLB characterized more significant decreases in plaque volume by stenting. Plaque redistribution to the proximal edge of the implanted stent occurred only in LLB. (Cardiol J 2020; 27, 3: 238-245)

Key words: plaque redistribution, stenting, intravascular ultrasound, near-infrared spectroscopy, stent edges

Address for correspondence: Tomasz Roleder, MD, PhD, Department of Cardiology, School of Health Sciences, Medical University of Silesia in Katowice, ul. Ziołowa 45/47, 40-001 Katowice, Poland, tel: +48 884096034, fax: +48 323598884, e-mail: tomaszroleder@gmail.com 


\section{Introduction}

Percutaneous coronary intervention (PCI) resolves ischemia by axial displacement of atherosclerotic plaque and increase of luminal area. However, the lipidic plaque is known to redistribute not only axially, but also longitudinally leading to tissue protrusion and stenosis at the stent edges. Both plaque protrusion [1,2] and reference vessel disease have been identified as predictors of poor outcome after PCI [3]. Intravascular imaging modalities like intravascular ultrasound (IVUS), optical coherence tomography (OCT) and nearinfrared spectroscopy (NIRS) provide insight into the morphology and composition of atherosclerotic plaque in vivo. OCT provides superior resolution, but its limited penetration depth, mainly through lipidic plaque, impairs the global assessment of the lesion, especially after stent implantation [4]. Due to superior penetration, IVUS overcomes this limitation of OCT, offering a global assessment of the lesion including the plaque structure behind the implanted stent [5]. Its combination with NIRS has augmented the capabilities of IVUS [6]. NIRS provides simplified algorithm of detection of lipid-rich lesions not only in atherosclerotic plaque but also in tissue located behind the implanted stent [6].

NIRS-IVUS studies demonstrated that lipidrich plaques might be prone to distal embolization after stenting because of redistribution of lipidic plaque and release of its debris into circulation [7-9]. Indeed, stenting of lipid-rich lesions increases the risk of periprocedural myocardial infarction suggesting that plaque redistribution is related to composition and morphology $[10,11]$. In the current study, the aim was to assess plaque redistribution after stent implantation by combined NIRS-IVUS imaging. It was hypothesized that the magnitude and geometry of plaque redistribution at the stent edges were related to its morphology and lipid content.

\section{Methods}

\section{Study design}

The present study was a prospective singlecenter study at the Medical University of Silesia in Katowice, Poland in which patients with stable coronary artery disease (SCAD) or non-ST-segment elevation-acute coronary syndrome (NSTE-ACS) were undergoing stent implantation with NIRS-IVUS. The investigators designed the trial and performed a retrospective analysis. Investigators assured data accuracy and collected source documents for adjudication. T.R. and M.D. performed intravascular imaging analysis, data management, and biostatistics. The institutional review board approved the study protocol. The study conformed to the Declaration of Helsinki and was approved by the Local Ethics Committee. All patients gave written informed consent.

\section{Participants}

Patients undergoing clinically indicated PCI for NSTE-ACS or SCAD using NIRS-IVUS guidance were screened. Patients with cardiogenic shock, New York Heart Association (NYHA) IV class heart failure, significant valvular heart disease, in-stent restenosis as the target lesion, reference vessel diameter less than $2.5 \mathrm{~mm}$, excessive tortuosity, pregnancy, hemophilia, renal failure (creatinine $>1.5 \mathrm{mg} / \mathrm{dL}$ ), and contrast allergy were excluded from the study.

\section{Procedures}

The current study employed combined NIRS-IVUS assessment of the target lesion pre- and post-stent implantation. All imaging was performed during the same procedure before predilatation or direct stenting, after stenting and final post-dilatation. The target lesion was selected at the operator's discretion after the diagnostic angiogram and fractional flow reserve assessement if needed. There were no complications related to NIRS-IVUS imaging, which was performed using heparin anticoagulation (activated clotting time $>300 \mathrm{~s}$ ) and following intracoronary nitroglycerine (100-200 $\mu \mathrm{m})$ administration. Combined NIRS and gray-scale IVUS image acquisition was performed using the commercially available TVC Imaging System ${ }^{\mathrm{TM}}$ and TVC Insight Catheter (InfraReDx, MA). The tip of the TVC catheter was positioned at least $10 \mathrm{~mm}$ distal to the imaging target lesion. Subsequently, the automated pullback was started at $0.5 \mathrm{~mm} / \mathrm{s}$ (240 rotations $/ \mathrm{min})$ until the TVC catheter entered the guiding catheter.

\section{NIRS images analysis}

NIRS map analysis allows the calculation of a lipid core burden index (LCBI). LCBI is estimated by dividing the number of yellow pixels per all pixels (without black ones) within the analyzed pullback length and are expressed per mill (\%o). The maximal LCBI was estimated in $4 \mathrm{~mm}$ pullback compartments for every analyzed segment pre- and post-stenting $\left(\operatorname{maxLCBI}_{4 \mathrm{~mm}}\right)$.

\section{Gray-scale IVUS image analysis}

The region of interest (ROI) was defined as the length of the artery covered by the stent preand post-procedure. Quantitative gray-scale IVUS 
measurements were performed every millimeter in scanned coronary segments pre and post-stenting. Cross-sectional images were quantified for lumen diameters and area, external elastic membrane (EEM) diameters and area, total plaque area, plaque burden, and lumen and EEM eccentricity. Additionally, after stenting, the stent area was estimated. The total plaque area was calculated as the difference between EEM area and lumen area (pre-stenting), or as a difference between EEM area and stent area (post-stenting). Plaque burden was calculated as total plaque area divided by EEM area $\times 100(\%)$. The IVUS reference lumen area was defined as the $4 \mathrm{~mm}$ located immediately proximal or distal to the ROI. The reference EEM area was calculated as an average of the proximal and distal EEM area. The remodeling index (RI) was calculated by dividing the EEM area at the minimal lumen area (MLA) by reference EEM area. Lesions with $\mathrm{RI} \leq 0.95$ were defined as negatively remodeled, while those with $\mathrm{RI} \geq 1.05$ were defined as positively remodeled. In every segment, a lumen vessel volume, EEM volume and stented volume (after stenting) was calculated based on the Simpson rule $\left[\mathrm{mm}^{3}\right]$. These data were used to estimate plaque volume (pre-stenting: EEM volume - vessel volume, post-stenting: EEM volume - stented volume $\left.\left[\mathrm{mm}^{3}\right]\right)$. All IVUS measurements were performed for the ROI and $1 \mathrm{~mm}$ and $4 \mathrm{~mm}$ long segments adjacent to the implanted stent (Fig. 1).

\section{Co-registration of NIRS-IVUS \\ pre- and post-stenting.}

During NIRS-IVUS pullback, anatomical landmarks were imprinted on the chemogram and bookmarked on the IVUS images - i.e., fiducial points, side branches, stent edges. Those landmarks allowed matching of NIRS-IVUS images to corresponding sections pre- and post-stenting on angiography.

\section{Lipid-rich lesions}

NIRS-IVUS defined high lipid burden lesions (HLB) as lesions with maxLCBI $_{4 \mathrm{~mm}}>265$ with positive RI [12]. Non-HLB segments were considered as lowlipid burden lesions (LLB). NIRS-IVUS data were analyzed off-line using CAAS intravascular software (Pie Medical Imaging BV). Coronary segments with incomplete and/or poor quality NIRS, IVUS scans were excluded from analysis (1 coronary segment, 1 patient).

\section{Statistical analysis}

Normality of the distribution of values was assessed using the Kolmogorov-Smirnov statistic,

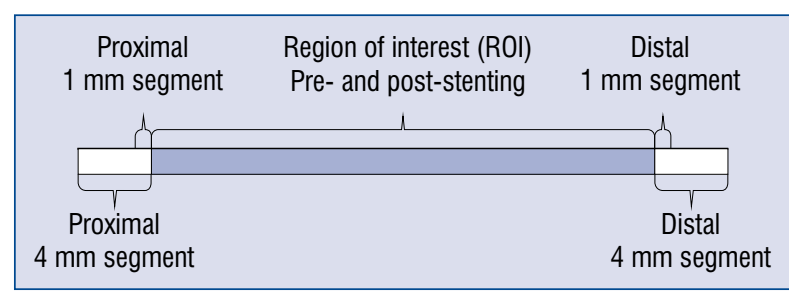

Figure 1. Intravascular ultrasound imaging measurements. The figure presents the analyzed segment by intravascular ultrasound imaging. The minimal lumen area and diameter external elastic lamina area and volume, lumen volume, plaque area volume, plaque burden and plaque eccentricity were measured for the region of interest, and for proximal and distal $1 \mathrm{~mm}$ and $4 \mathrm{~mm}$ long segments adjacent to the stent. All measurements were performed before and after stenting.

and homogeneity of variances assessed using the Levene test. For normally distributed values, data are presented as mean with standard deviation (SD), for non-normally distributed values data are presented as median with interquartile range (IQR, 25 percentile, 75 percentile). Normally distributed data were compared using a paired t-test, and nonnormally distributed data were compared using the Mann-Whitney-test. The categorical data were compared using the Fisher exact test or $\chi^{2}$ test. A two-tailed p-value of 0.05 was considered as statistically significant. Data analysis was performed using Medcalc software version 17.1.

\section{Results}

\section{Study group}

Between September 2015 and August 2016 intravascular imaging was performed in 50 stents implanted in 49 patients with either SCAD $(\mathrm{n}=33 ; 67 \%)$ or NSTE-ACS syndromes $(\mathrm{n}=16$; $33 \%)$. HLB lesions were identified in 9 patients, and LLB lesions were detected in 40 patients (50 ROIs). HLB patients were characterized by a higher level of total cholesterol and triglycerides, and trend towards a higher prevalence of diabetes. Baseline clinical characteristics are presented in Table 1.

\section{Angiographic data analysis}

and procedure details

There were now differences in the location of HLB lesions and LLB lesions - left anterior descending artery: $5(56 \%)$ vs. $19(46 \%)$, circumflex artery: 3 (33\%) vs. 10 (24\%), ramus intermedius: 0 vs. $1(2 \%)$, left main: 0 vs. $1(2 \%)$, right coronary artery: $1(11 \%)$ vs. $10(24 \%), \mathrm{p}=0.846$. Two HLB lesions (22\%) and 14 (34\%) LLB lesions were lo- 
Table. 1. Patient characteristics.

\begin{tabular}{|c|c|c|c|}
\hline & $\begin{array}{l}\text { Patients with low lipid } \\
\text { burden lesions }(n=40)\end{array}$ & $\begin{array}{l}\text { Patients with high lipid } \\
\text { burden lesions }(\mathrm{n}=9)\end{array}$ & $\mathbf{P}$ \\
\hline Age [years] & $66.17 \pm 11.32$ & $65.5 \pm 10.72$ & 0.920 \\
\hline Male gender & $32(80 \%)$ & $5(55 \%)$ & 0.113 \\
\hline Body mass index $\left[\mathrm{kg} / \mathrm{m}^{2}\right]$ & 26.7 (IQR 25.23, 27.93) & 25.15 (IQR 20.70, 36.09) & 0.771 \\
\hline NSTEMI/UA/SCAD & $4(10 \%) / 20(50 \%) / 16(30 \%)$ & $0 / 5(55 \%) / 4(45 \%)$ & 0.433 \\
\hline \multicolumn{4}{|l|}{ Risk factors } \\
\hline Hypertension & $36(90 \%)$ & $8(88 \%)$ & 0.634 \\
\hline Hyperlipidemia & $40(100 \%)$ & $9(100 \%)$ & - \\
\hline Diabetes mellitus & $11(27 \%)$ & $6(67 \%)$ & 0.058 \\
\hline Current smoking & $3(7 \%)$ & $1(11 \%)$ & 0.714 \\
\hline \multicolumn{4}{|l|}{ Pharmacological therapy } \\
\hline Acetylsalicylic acid & $38(95 \%)$ & $8(88 \%)$ & 0.765 \\
\hline Thienopyridine & $27(67 \%)$ & $3(33 \%)$ & 0.153 \\
\hline Beta-adrenergic antagonist & $32(80 \%)$ & $6(67 \%)$ & 0.519 \\
\hline Calcium channel antagonist & $10(25 \%)$ & $5(56 \%)$ & 0.148 \\
\hline ARB/ACEI & $27(67 \%)$ & $6(67 \%)$ & 0.732 \\
\hline Statin & $36(90 \%)$ & $9(100 \%)$ & 0.623 \\
\hline Other lipid-lowering therapy & $4(10 \%)$ & $2(22 \%)$ & 0.654 \\
\hline Oral antidiabetics & $10(25 \%)$ & $5(56 \%)$ & 0.148 \\
\hline Insulin & $1(2 \%)$ & $1(11 \%)$ & 0.792 \\
\hline \multicolumn{4}{|l|}{ Laboratory results } \\
\hline Total cholesterol [mg/dL] & 139 (IQR 127, 153) & 171 (IOR 146.92, 244.64) & 0.031 \\
\hline LDL cholesterol [mg/dL] & 76 (IOR 69, 93) & 99.00 (IOR 69.69, 132.03) & 0.120 \\
\hline HDL cholesterol [mg/dL] & 40.00 (IOR $36.76,46.00)$ & 44.50 (IOR 38.67, 50.16) & 0.401 \\
\hline Triglyceride [mg/dL] & 102.02 (IQR 91.58, 115.19) & 154.50 (IOR 98.10, 355.03) & 0.012 \\
\hline $\mathrm{GRF}\left[\mathrm{mL} / \mathrm{min} / 1.73 \mathrm{~m}^{2}\right]$ & $72.40 \pm 15.93$ & $67.62 \pm 25.84$ & 0.473 \\
\hline
\end{tabular}

NSTEMI — non-ST-segment elevation myocardial infarction, UA — unstable angina, SCAD — stable coronary artery disease, ARB — angiotensin II receptor blocker, ACEI — angiotensin-converting-enzyme inhibitor; LDL — low density lipoprotein, HDL — high-density lipoprotein, GRF — glomerular filtration rate; IQR — interquartile range

cated in proximal segments of the coronary artery $(\mathrm{p}=0.379)$.

There were also no differences in type of drug eluting stents implanted in HLB and LLB lesions - everolimus eluting: 7 (78\%) vs. 21 (51\%), sirolimus eluting $2(22 \%)$ vs. $11(27 \%)$, biolimus eluting 0 vs. $9(22 \%) ; \mathrm{p}=0.106$. There were no differences in the stent diameter $[\mathrm{mm}]-3(3,3.5)$ vs. $3(3,3.5), \mathrm{p}=0.250$ and stent length $[\mathrm{mm}]-$ $15(12,24)$ vs. $22(18,28), p=0.306$ implanted in HLB and LLB lesions.

\section{Plaque modificationof the stented segment}

Both HLB and LLB lesions were characterized by an increase of minimal lumen diameter (MLD), minimal lumen area (MLA), lumen volume and EEM volume and a decrease of maxLCBI ${ }_{4 \mathrm{~mm}}$, total plaque area, plaque burden and plaque volume after stenting. Although the plaque volume decreased in both HLB and LLB lesions, the change was significantly higher in HLB lesions (Table 2, Fig. 2). Stenting increased remodeling index only in LLB lesions, having no impact on the RI in HLB lesions. The observed differences in plaque volume correlated with maxLCBI $\mathrm{mm}_{4 \mathrm{~m}}$ before stenting $(\mathrm{r}=0.48 ; \mathrm{p}<0.01)$ in all 50 lesions (Fig. 2). NIRS-IVUS characteristics of stented lesions is presented in Table 2. Representative images of plaque modification are presented in Figure 3 and Supplementary Figures 1 and 2.

Assessment of the proximal segment adjacent to the stent after the procedure

In $4 \mathrm{~mm}$ proximal segment adjacent to stent, maxLCBI $\mathrm{mm}_{4 \mathrm{~mm}}$ decreased after stenting in LLB lesions $-34(0,207)$ vs. $0(0,45), \mathrm{p}=0.049$. However, plaque 
Table 2. Near-infrared spectroscopy and intravascular ultrasound results of the stented segment.

\begin{tabular}{|c|c|c|c|c|c|c|}
\hline \multirow[t]{2}{*}{ Parameters } & \multicolumn{3}{|c|}{ Low lipid burden lesions ( $n=41$ ) } & \multicolumn{3}{|c|}{ High lipid burden lesions ( $n=9$ ) } \\
\hline & Pre-stenting & Post-stenting & $\mathbf{P}$ & Pre-stenting & Post-stenting & $\mathbf{P}$ \\
\hline \multicolumn{7}{|l|}{ Stented segment } \\
\hline $\mathrm{ROI}[\mathrm{mm}]$ & $21.98 \pm 9.01$ & $21.96 \pm 8.98$ & 0.804 & $25.71 \pm 15.38$ & $25.70 \pm 15.39$ & 0.346 \\
\hline MLD [mm] & $1.64 \pm 0.31$ & $2.23 \pm 0.37$ & $<0.001$ & $1.53 \pm 0.13$ & $2.28 \pm 0.34$ & $<0.001$ \\
\hline $\mathrm{MLA}\left[\mathrm{mm}^{2}\right]$ & $2.65 \pm 1.21$ & $5.31 \pm 1.68$ & $<0.001$ & $2.94 \pm 1.40$ & $6.15 \pm 1.39$ & $<0.001$ \\
\hline $\begin{array}{l}\text { Stenosis (MLA) } \\
\text { on reference [\%] }\end{array}$ & $55.36 \pm 13.01$ & $17.90 \pm 14.38$ & $<0.001$ & $57.63 \pm 10.19$ & $19.00 \pm 14.78$ & 0.006 \\
\hline Lumen volume $\left[\mathrm{mm}^{3}\right]$ & $\begin{array}{c}98.80 \\
(63.15,131.45)\end{array}$ & $\begin{array}{c}128.30 \\
(86.97,185.37)\end{array}$ & $<0.001$ & $\begin{array}{c}96.70 \\
(69.10,112.62)\end{array}$ & $\begin{array}{c}181.30 \\
(76.22,266.37)\end{array}$ & 0.004 \\
\hline EEM volume $\left[\mathrm{mm}^{3}\right]$ & $\begin{array}{c}226.70 \\
(140.77,315.82)\end{array}$ & $\begin{array}{c}288.60 \\
(169.42,377.62)\end{array}$ & $<0.001$ & $\begin{array}{c}244.40 \\
(149.55,379.10)\end{array}$ & $\begin{array}{c}317.30 \\
(144.47,535.52)\end{array}$ & 0.012 \\
\hline$\Delta \mathrm{EEM}$ volume $\left[\mathrm{mm}^{3}\right]$ & $\begin{array}{c}46.45 \\
(21.50,74.85)\end{array}$ & & & $\begin{array}{c}67.40 \\
(16.22,116.62)\end{array}$ & & 0.423 \\
\hline Plaque volume $\left[\mathrm{mm}^{3}\right]$ & $\begin{array}{c}124.50 \\
(68.00,186.20)\end{array}$ & $\begin{array}{c}101.10 \\
(67.87,165.95)\end{array}$ & $<0.001$ & $\begin{array}{c}144.70 \\
(80.47,274.25)\end{array}$ & $\begin{array}{c}97.60 \\
(56.82,223.45)\end{array}$ & 0.004 \\
\hline$\triangle \mathrm{MPV}\left[\mathrm{mm}^{3}\right]$ & $13.50(1.50,28.84)$ & & & $28.00(22.60,57.10)$ & & 0.019 \\
\hline $\operatorname{maxLCBI4}[\mathrm{mm}]$ & $339.07 \pm 268.22$ & $124.60 \pm 160.96$ & $<0.001$ & $564.11 \pm 166.82$ & $258.11 \pm 234.24$ & 0.004 \\
\hline EEM area $\left[\mathrm{mm}^{2}\right]$ & $\begin{array}{c}10.00 \\
(7.50,11.60)\end{array}$ & $\begin{array}{c}11.30 \\
(9.27,13.25)\end{array}$ & $<0.001$ & $\begin{array}{c}12.70 \\
(12.10,13.47)\end{array}$ & $\begin{array}{c}13.10 \\
(11.72,16.20)\end{array}$ & 0.820 \\
\hline EEM eccentricity & $0.09(0.07,0.15)$ & $0.09(0.06,0.13)$ & 0.551 & $0.09 \pm 0.01$ & $0.11 \pm 0.04$ & 0.341 \\
\hline Lumen eccentricity & $0.09(0.06,0.14)$ & $0.13(0.08,0.18)$ & 0.038 & $0.17 \pm 1.12$ & $0.20 \pm 0.10$ & 0.181 \\
\hline Total plaque area $\left[\mathrm{mm}^{2}\right]$ & $7.4(5.12,8.62)$ & $4.7(3.67,6.40)$ & $<0.001$ & $11.51 \pm 5.01$ & $5.72 \pm 1.80$ & 0.009 \\
\hline Plaque burden [\%] & $72.19 \pm 8.12$ & $55.03 \pm 6.87$ & $<0.001$ & $79.26 \pm 8.57$ & $53.0 \pm 9.9$ & $<0.001$ \\
\hline Remodeling index & $0.87 \pm 0.23$ & $1.09 \pm 0.27$ & $<0.001$ & $1.23 \pm 0.11$ & $1.20 \pm 0.25$ & 0.739 \\
\hline
\end{tabular}

$\mathrm{ROI}$ - region of interest; MLD - minimal lumen diameter; MLA - minimal lumen area; EEM — external elastic lamina; maxLCBI4 mm maximal lipid core burden index in four millimeters; $\triangle \mathrm{MPV}$ - delta plaque volume

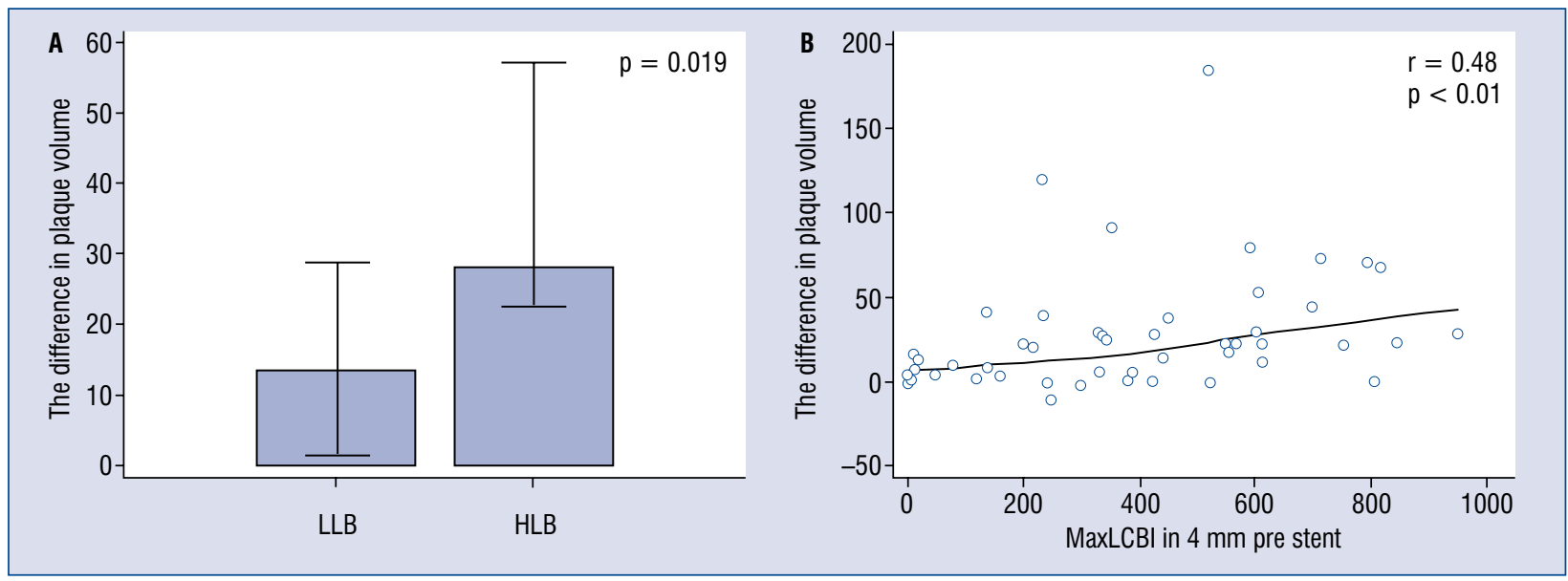

Figure 2. Plaque volume pre- and post-stenting and lipid core burden index (LCBI) values. A. The difference in plaque volume for near-infrared spectroscopy and intravascular ultrasound (NIRS-IVUS) high lipid burden lesions (HLB, $\operatorname{maxLCBI}_{4 \mathrm{~mm}}>265$ and positive vessel remodeling) and for NIRS-IVUS low lipid burden (LLB) lesions; B. The correlation between the difference in plaque volume pre- and post-stenting and $\operatorname{maxLCBI}_{4 \mathrm{~mm}}$ values in all lesions.

burden [\%] - $45.48(40.34,51.55)$ vs. $51.75(47.48$, $55.76), \mathrm{p}=0.030$ and total plaque area $\left[\mathrm{mm}^{2}\right]-6.30$ $(5.17,7.85)$ vs. $7.05(6.08,8.43), \mathrm{p}=0.005$ increased in the first millimeter adjacent to the implanted stent in LLB lesions. Such plaque modification was not observed in HLB lesions. NIRS-IVUS characteristics of proximally segment adjacent to the implanted stent is presented in Supplementary Table 1. 


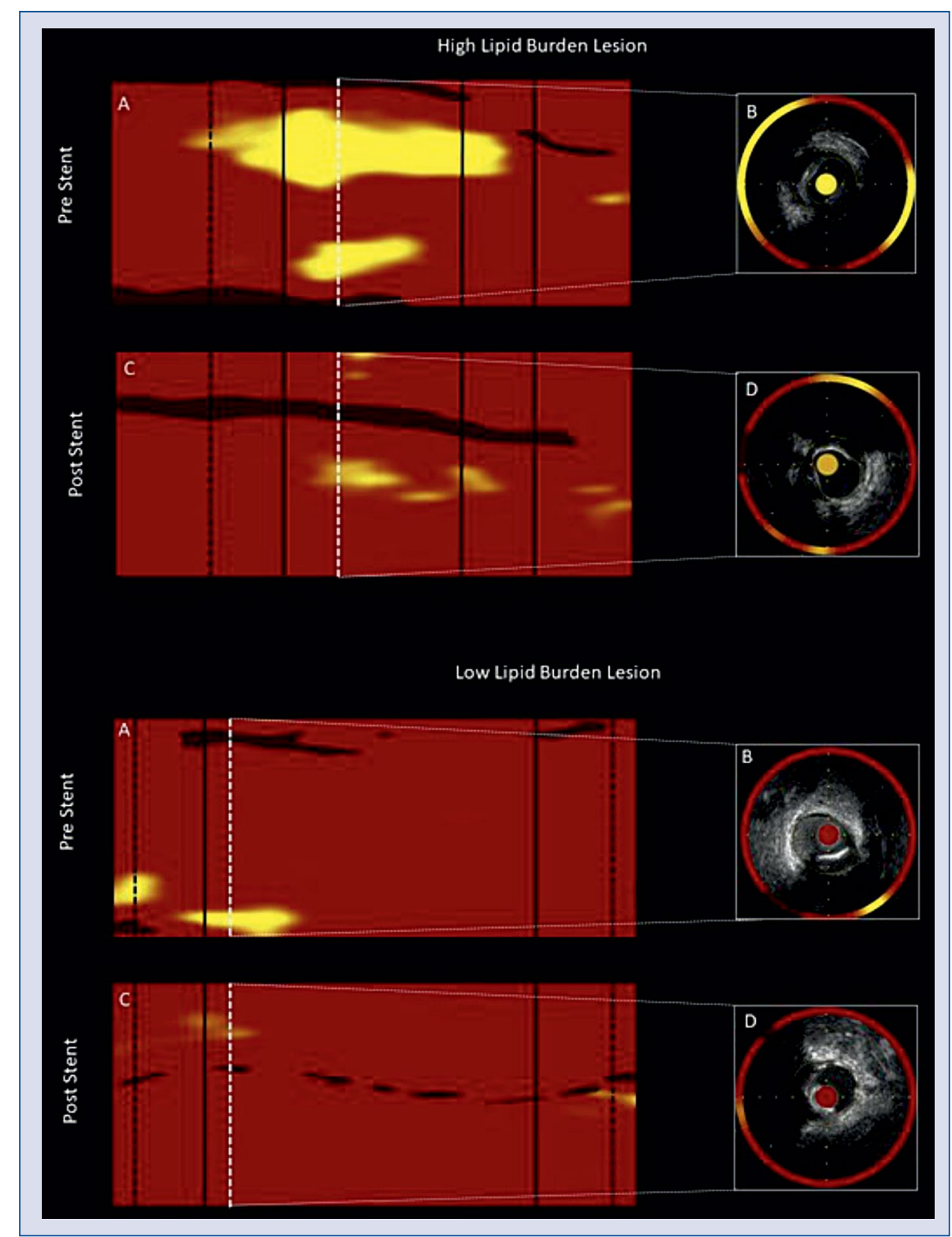

Figure 3. Representative image of near-infrared spectroscopy and intravascular ultrasound (NIRS-IVUS) imaging pre- and post-stenting of high lipid burden and low lipid burden lesions; A, C. NIRS maps pre- and post-stent implantation. Black lines indicate stent edges. Black dashed lines limit $4 \mathrm{~mm}$ proximal and distal segments adjacent to the stent. White dashed lines indicate the NIRS-IVUS cross-section image. B, D. NIRS-IVUS cross-sectional image of minimal lumen area (MLA), external elastic lamina (EEM) and stent contours. High lipid burden lesion: A. Pre-stenting $\operatorname{maxLCBI}{ }_{4 \mathrm{~mm}}=548 ;$ C. Post-stenting $\operatorname{maxLCBI}_{4 \mathrm{~mm}}=202 ;$ B. Pre-stenting: $\mathrm{MLA}=3.8 \mathrm{~mm}^{2}$, plaque burden $=71 \%$, plaque area $=9.2 \mathrm{~mm}^{2}$, EEM area $=12.9 \mathrm{~mm}^{2}$, remodeling index $=1.34 ;$ D. Post-stenting: MLA $=6.4 \mathrm{~mm}^{2}$, plaque burden $=48.8 \%$, plaque area $=6.1 \mathrm{~mm}^{2}$, EEM area $=12.4 \mathrm{~mm}^{2}$, stent area $=5.0 \mathrm{~mm}^{2}$, remodeling index $=1.22$; delta mean plaque volume $=22.9 \mathrm{~mm}^{3}$. Low lipid burden lesion: $A$. Pre-stenting $\mathrm{maxLCBI}_{4 \mathrm{~mm}}$ $=137 ;$ C. Post-stenting $\operatorname{maxLCBI}{ }_{4 m}=30 ;$ B. Pre-stenting: $\mathrm{MLA}=3.5 \mathrm{~mm}^{2}$, plaque burden $=61 \%$, plaque area $=6.4 \mathrm{~mm}^{2}$, EEM area $=10.6 \mathrm{~mm}^{2}$, remodeling index $=0.72 ; \mathrm{D}$. Post-stenting: $\mathrm{MLA}=5.8 \mathrm{~mm}^{2}$, plaque burden $=53 \%$, plaque area $=6.4 \mathrm{~mm}^{2}$, EEM area $=12.0 \mathrm{~mm}^{2}$, stent area $=6.6 \mathrm{~mm}^{2}$, remodeling index $=1.29 ;$ delta mean plaque volume $=8.2 \mathrm{~mm}^{3}$.

\section{Assessment of the distal segment adjacent} to the stent after the procedure

After the procedure, there were no differences in maxLCBI ${ }_{4 \mathrm{~mm}}$, plaque burden, plaque volume in distal
$4 \mathrm{~mm}$ segment adjacent to the implanted stent in both HLB and LLB lesions. NIRS-IVUS characteristics of the segment located distally to the implanted stent is presented in Supplementary Table 2. 


\section{Discussion}

This study expands previous observations from intravascular imaging on plaque redistribution and lipid burden following stenting, using NIRS-IVUS [13]. It is confirmed herein, that plaque redistribution caused by stenting is related to its composition. Lipid-rich lesions characterized a more significant decrease in plaque volume after stenting, especially in HLB. Interestingly, plaque redistribution to the proximal edges of the stent occurred only in LLB.

Initial IVUS studies conducted in patients with SCAD demonstrated that stent implantation redistributed plaque longitudinally across the vessel to the proximal and distal edges of the stent with the potential for release of plaque debris into coronary circulation [7-9]. NIRS imaging alone confirmed a lipidic component of these findings [14]. Combined NIRS-IVUS imaging has demonstrated that a decrease in LCBI with a reduction in plaque volume led to edge redistribution of lipidic plaque in ST-segment elevation myocardial infarction (STEMI) patients [13]. The current study of non-STEMI patients and SCAD patients confirms that stenting of HLB lesions is associated with a decrease in plaque volume.

Interestingly, plaque shift to the proximal stent edge was found only in LLB lesions. The smaller lumen volume after stenting LLB suggest that these plaques have a smaller potential to be compressed and are less likely to protrude through stent struts. Thus it may affect final stent expansion and prone redistribution of LLB plaques to the edges of implanted stent edges to make space for the stent. Moreover, as it was shown a correlation between HLB lesions and thin fibrous cap atheroma (TFCA) $[12,15]$, it is also possible that LLB lesions are less prone to embolization into the microcirculation from a thick cap. Indeed, OCT studies have shown that TFCA correlates with type IVa myocardial infarction after PCI [14], and OCT defined TFCA is a strong predictor of periprocedural infarction [10]. Taken together the present results suggest that both lipid-rich plaque and distinct plaque morphology may be necessary to trigger distal embolization [16]. Previous studies have shown that the use of aggressive lipid-lowering therapy can decrease lipid core and increase fibrous cap thickness [17, 18]. The routine use of high-dose statins before planned stent implantation to reduce no-reflow phenomenon requires further attention [19].

The Color Registry showed previously that lesions with maxLCBI ${ }_{4 \mathrm{~mm}}>500$ had an increased risk of periprocedural myocardial infarction [11].
The findings prompted the CANARY trial, which tested the potential benefit of distal protection during PCI for lesions with maxLCBI ${ }_{4 \mathrm{~mm}}>600$. The trial failed to show a benefit, perhaps due to the inherent morbidity of filter placement but was also stopped prematurely due to difficulties in identifying patients suitable for randomization [20]. The present data suggests that plaque morphology assessment should also be applied to assess the risk of distal embolization.

The current study showed that LLB lesions had a smaller decrease in plaque volume with an increase in plaque area and plaque burden at the proximal edge of the implanted stent, but not at the distal edge. It is possible that this is a result of the difference in size of the vessel proximally versus distally. The relatively large proximal vessel size may be able to accommodate plaque shift to a greater extent than the distal edge. Since NIRS cannot determine depth (axial dimension of the lipid), a decreased lipid length could result in decreased LCBI values but not an actual reduction in lipid burden, rather indicating an axial accumulation [21]. Nevertheless, the plaque shift to the proximal edge of the stent advocates use of the "red to red" (healthy to healthy segment) stenting strategy to reduce adverse clinical outcomes [22].

\section{Limitations of the study}

The study has several limitations. A small group of patients were included in the study, and both acute and stable coronary patients were assessed. The HLB lesion definition relied on previously presented NIRS-IVUS data against OCT [12]. The addition of OCT imaging to NIRS-IVUS imaging could have improved the accuracy of HLB detection in this study. Although there was no strict protocol for stent implantation, it does represent real-world practice.

\section{Conclusions}

NIRS-IVUS HLB lesions had a more significant decrease in plaque volume by stenting without plaque distribution to the edges of the stent. Plaque redistribution to the proximal edge of the implanted stent was observed only in LLB lesions.

\section{Funding}

The study was supported by the statutory funds of the Medical University of Silesia in Katowice, Poland.

Conflict of interest: None declared 


\section{References}

1. Prati F, Romagnoli E, Gatto L, et al. Clinical Impact of Suboptimal Stenting and Residual Intrastent Plaque/Thrombus Protrusion in Patients With Acute Coronary Syndrome: The CLI-OPCI ACS Substudy (Centro per la Lotta Contro L'Infarto-Optimization of Percutaneous Coronary Intervention in Acute Coronary Syndrome). Circ Cardiovasc Interv. 2016; 9(12), doi: 10.1161/CIRCINTERVENTIONS.115.003726, indexed in Pubmed: 27965297.

2. Soeda T, Uemura S, Park SJ, et al. Incidence and clinical significance of poststent optical coherence tomography findings: one-year follow-up study from a multicenter registry. Circulation. 2015; 132(11): 1020-1029, doi: 10.1161/CIRCULATIONAHA.114.014704, indexed in Pubmed: 26162917.

3. Prati F, Romagnoli E, Burzotta F, et al. Clinical Impact of OCT Findings During PCI: The CLI-OPCI II Study. JACC Cardiovasc Imaging. 2015; 8(11): 1297-1305, doi: 10.1016/j. jcmg.2015.08.013, indexed in Pubmed: 26563859.

4. Jang IK, Bouma B, Kang DH, et al. Visualization of coronary atherosclerotic plaques in patients using optical coherence tomography: comparison with intravascular ultrasound. J Am Coll Cardiol. 2002; 39(4): 604-609, doi: 10.1016/s0735-1097(01)01799-5.

5. Yock PG, Linker DT. Intravascular ultrasound. Looking below the surface of vascular disease. Circulation. 1990; 81(5): 1715-1718, indexed in Pubmed: 2184950.

6. Ali ZA, Roleder T, Narula J, et al. Increased thin-cap neoatheroma and periprocedural myocardial infarction in drug-eluting stent restenosis: multimodality intravascular imaging of drug-eluting and bare-metal stents. Circ Cardiovasc Interv. 2013; 6(5): 507-517, doi: 10.1161/CIRCINTERVENTIONS.112.000248, indexed in Pubmed: 24065447.

7. Ahmed JM, Mintz GS, Weissman NJ, et al. Mechanism of lumen enlargement during intracoronary stent implantation: an intravascular ultrasound study. Circulation. 2000; 102(1): 7-10, indexed in Pubmed: 10880407.

8. Algowhary M, Matsumura A, Hashimoto Y, et al. Poststenting axial redistribution of atherosclerotic plaque into the reference segments and lumen reduction at the stent edge. Int Heart J. 2006; 47(2): 159-171, doi: 10.1536/ihj.47.159.

9. Maehara A, Takagi A, Okura $\mathrm{H}$, et al. Longitudinal plaque redistribution during stent expansion. Am J Cardiol. 2000; 86(10): 1069-1072, indexed in Pubmed: 11074201.

10. Kini AS, Motoyama S, Vengrenyuk Y, et al. Multimodality Intravascular Imaging to Predict Periprocedural Myocardial Infarction During Percutaneous Coronary Intervention. JACC Cardiovasc Interv. 2015; 8(7): 937-945, doi: 10.1016/j.jcin.2015.03.016, indexed in Pubmed: 26088511.

11. Goldstein JA, Maini B, Dixon SR, et al. Detection of lipid-core plaques by intracoronary near-infrared spectroscopy identifies high risk of periprocedural myocardial infarction. Circ Cardiovasc Interv. 2011; 4(5): 429-437, doi: 10.1161/CIRCINTERVENTIONS.111.963264, indexed in Pubmed: 21972399.

12. Roleder T, Kovacic JC, Ali Z, et al. Combined NIRS and IVUS imaging detects vulnerable plaque using a single catheter sys- tem: a head-to-head comparison with OCT. EuroIntervention. 2014; 10(3): 303-311, doi: 10.4244/EIJV10I3A53, indexed in Pubmed: 24769522.

13. Noori M, Thayssen P, Veien KT, et al. Lipid-core burden response to stent implantation assessed with near-infrared spectroscopy and intravascular ultrasound evaluation in patients with myocardial infarction. Cardiovasc Revasc Med. 2017; 18(3): 182-189, doi: 10.1016/j.carrev.2016.12.018, indexed in Pubmed: 28109718.

14. Porto I, Di Vito L, Burzotta F, et al. Predictors of periprocedural (type IVa) myocardial infarction, as assessed by frequency-domain optical coherence tomography. Circ Cardiovasc Interv. 2012; 5(1): 89-96, S1, doi: 10.1161/CIRCINTERVENTIONS.111.965624, indexed in Pubmed: 22298799.

15. Biały D, Wawrzyńska M, Arkowski J, et al. Multimodality imaging of intermediate lesions: Data from fractional flow reserve, optical coherence tomography, near-infrared spectroscopy-intravascular ultrasound. Cardiol J. 2018; 25(2): 196-202, doi: 10.5603/ /CJ.a2017.0082, indexed in Pubmed: 28714527.

16. Wolny R, Dębski A, Kruk M, et al. Slow-flow phenomenon after elective percutaneous coronary intervention of computed tomography-detected vulnerable coronary lesion. Postepy Kardiol Interwencyjnej. 2014; 10(3): 181-184, doi: 10.5114/ /pwki.2014.45145, indexed in Pubmed: 25489304.

17. Nishio R, Shinke T, Otake H, et al. Stabilizing effect of combined eicosapentaenoic acid and statin therapy on coronary thin-cap fibroatheroma. Atherosclerosis. 2014; 234(1): 114-119, doi: 10.1016/j.atherosclerosis.2014.02.025, indexed in Pubmed: 24637411.

18. Chia S, Raffel OC, Takano M, et al. Association of statin therapy with reduced coronary plaque rupture: an optical coherence tomography study. Coron Artery Dis. 2008; 19(4): $237-$ -242, doi: 10.1097/MCA.0b013e32830042a8, indexed in Pubmed: 18480667 .

19. Zhao JL, Yang YJ, Pei WD, et al. The effect of statins on the noreflow phenomenon. Am J Cardiovasc Drugs. 2012; 9(2): 81-89, doi: 10.1007/bf03256579.

20. Stone GW, Maehara A, Muller JE, et al. CANARY Investigators. Plaque Characterization to Inform the Prediction and Prevention of Periprocedural Myocardial Infarction During Percutaneous Coronary Intervention: The CANARY Trial (Coronary Assessment by Near-infrared of Atherosclerotic Rupture-prone Yellow). JACC Cardiovasc Interv. 2015; 8(7): 927-936, doi: 10.1016/j. jcin.2015.01.032, indexed in Pubmed: 26003018.

21. Gardner CM, Tan H, Hull EL, et al. Detection of lipid core coronary plaques in autopsy specimens with a novel catheter-based near-infrared spectroscopy system. JACC Cardiovasc Imaging. 2008; 1(5): 638-648, doi: 10.1016/j.jcmg.2008.06.001, indexed in Pubmed: 19356494

22. Waxman S, Freilich MI, Suter MJ, et al. A case of lipid core plaque progression and rupture at the edge of a coronary stent: elucidating the mechanisms of drug-eluting stent failure. Circ Cardiovasc Interv. 2010; 3(2): 193-196, doi: 10.1161/CIRCINTERVENTIONS.109.917955, indexed in Pubmed: 20407116. 